\title{
First observation of E1 transitions from the octupole band to the excited $\mathrm{O}_{2}^{+}$pairing isomer band in the rare earth nucleus ${ }^{154} \mathrm{Dy}$
}

G. L. Zimba ${ }^{* a, b}$, S. P. Bvumbi ${ }^{a}$, L. P. Masiteng ${ }^{a}$, P. Jones $^{b}$, J. F. Sharpey-Schafer ${ }^{b, d}$, E. Lawrie $^{b}$, S. N. T. Majola ${ }^{b, c}$, T. S. Dinoko ${ }^{b}$, J. J. Lawrie ${ }^{b}$, D. Roux ${ }^{f}$, P. Papka ${ }^{e}$, J. E. Easton $^{d}$, D. Negi ${ }^{b}$, O. Shirinda ${ }^{b}$, and N. Khumalo ${ }^{d}$

${ }^{a}$ University of Johannesburg,

Physics Department, P.O Box 524, Auckland Park 2006, South Africa.

${ }^{b}$ iThemba Laboratory for Accelerator Based Sciences

P.O Box 722, Somerset-West, ZA-7129 South Africa.

${ }^{c}$ University of Cape Town,

Physics Department Private Bag X3, Rondebosch 7701, South Africa.

${ }^{d}$ University of Western Cape,

Physics Department, P/B X17, Bellville, ZA7535 South Africa.

${ }^{e}$ Stellenbosch University,

Physics Department, P/B X1, Matieland, 7602, South Africa.

${ }^{f}$ Rhodes University,

Physics Department, P.O Box 94, Grahamstown 6140, South Africa.

E-mail: zimbageo@yahoo.com

The low spin states of ${ }^{154}$ Dy were studied with the AFRODITE spectrometer array [1] equipped with 9 clover High Purity Germanium (HPGe) detectors with Compton suppressing Bismuth Germanium (BGO) shielding at iThemba LABS. The Fusion Evaporation reaction ${ }^{155} \mathrm{Gd}\left({ }^{3} \mathrm{He}\right.$, 4n) ${ }^{154} \mathrm{Dy}$ at $37.5 \mathrm{MeV}$ was used to populate low spin states. A first observation of enhanced E1 transitions in the transitional $\mathrm{N}=88$ isotones ${ }^{150} \mathrm{Sm}$ and ${ }^{152} \mathrm{Gd}$ from the levels in the first excited $0^{+}$band to the lowest negative parity band [2]. The results we report here are on the first observation of enhanced E1 transitions from the low-lying negative parity band to the band built on the first excited $0^{+}$state in ${ }^{154} \mathrm{Dy}$. These measurements on ${ }^{154} \mathrm{Dy}$ extend the systematics on the relationship between the first excited positive parity pairing isomer band and the first excited negative parity band as the nuclear deformation decreases with increasing proton number.

53rd International Winter Meeting on Nuclear Physics,

26-30 January 2015

Bormio, Italy

${ }^{*}$ Speaker. 


\section{Introduction}

The Dysprosium ( ${ }^{154} \mathrm{Dy}$ ) nucleus with 6 neutrons and 2 protons outside the closed shell offers a great opportunity to study the octupole deformation in $\mathrm{N}=88$ nuclei. ${ }^{154} \mathrm{Dy}$ is a transition nucleus, thus it rapidly changing shape from spherical to quadrupole deformation. This is illustrated in experimental measurements of $4^{+}$and $2^{+}$states [3], see Fig. 1.

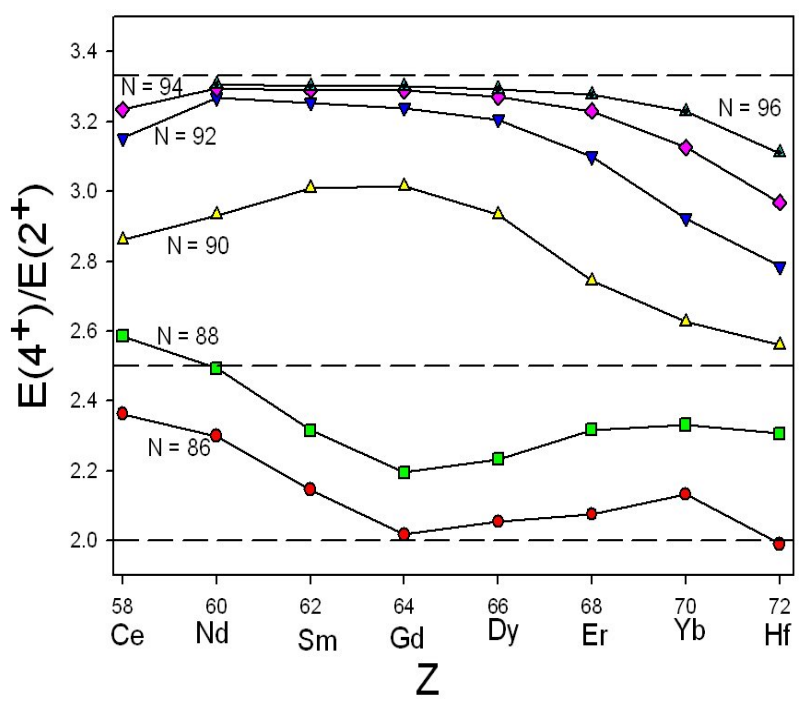

Figure 1: $\frac{E\left(4^{+}\right)}{E\left(2^{+}\right)}$energy-ratio systematics for even-even nuclei as a function of atomic number Z. The horizontal dashed lines represent limits expected for pure vibrational, rotational, and $\gamma$-soft behavior, respectively [3].

Nuclei with quadrupole and octupole deformations are known to possess two rotational bands having positive and negative parities. The lowest lying negative parity bands are usually built on $3^{-}$or $1^{-}$states are referred to as the octupole bands. Due to missing E2 in-band transitions in these states, recent assumptions that structures associated with octupole excitations in rare earth region are associated with tetrahedral symmetries [4]. One of the features of octupole deformation in nuclei such as ${ }^{154} \mathrm{Dy}$ is the presence of E3 transitions from the ground state band to the octupole band. Spear and Catford [5] showed that there a maximum in strength of E3 transitions for $\mathrm{N}=$ 88 and $\mathrm{Z}=62$ nuclei. The other feature of octupole deformation is the presence of E1 transitions between the first excited $0^{+}$band and the lowest lying negative parity. E1 transitions have been observed between the first excited $0^{+}$band and the lowest lying negative parity band in experiments studying the structures of other $\mathrm{N}=88$ isotones by Bvumbi [2]. The octupole structure has been descried and explained as due to the nearness of $\Delta J^{\pi}=3^{-}$shell model orbits to the Fermi surface [6]. For $\mathrm{N}=88$ isotones these orbitals are the $i_{13 / 2}-f_{7 / 2}$ neutron orbits and $h_{11 / 2}-d_{5 / 2}$ proton orbits respectively.

\section{Experimental and data analysis procedure}

Low spin excited states in ${ }^{154}$ Dy were populated via the reaction ${ }^{155} \mathrm{Gd}\left({ }^{3} \mathrm{He}, 4 \mathrm{n}\right)$ with a 37.5 $\mathrm{MeV}$ beam from the separator sector cyclotron at iThemba Labs. Self-supporting $3.2 \mathrm{mg} . \mathrm{cm}^{-2}$ 
target was used; therefore no Doppler correction was necessary since the target thickness was sufficient to stop recoiling residual nuclei. The $\gamma$ rays emitted from the excited ${ }^{154}$ Dy were detected with the AFRODITE spectrometer array [1] which consists of nine Compton suppressed HPGe clover detectors. The trigger condition was set to accept events when at least two or more clover detectors fired in coincidence. A total of $1.72 \times 10^{9}$ events were collected over two and half days of beam time which enabled us to unfold $\gamma^{2}$ events into two-dimensional matrix using MTsort [7].

The level scheme for ${ }^{154}$ Dy was deduced from the matrix which contained $\gamma-\gamma$ coincidence data using Radware software packages [8]. The ordering of the transitions was based on $\gamma-\gamma$ coincidence relationships. In order to assign correct spin and parity to the newly observed transitions, we obtained $\gamma$ multipolarities by using Linear Polarization Anisotropy (LPA) [9] and Directional Correlation from Oriented states (DCO).

\section{Results and discussion}

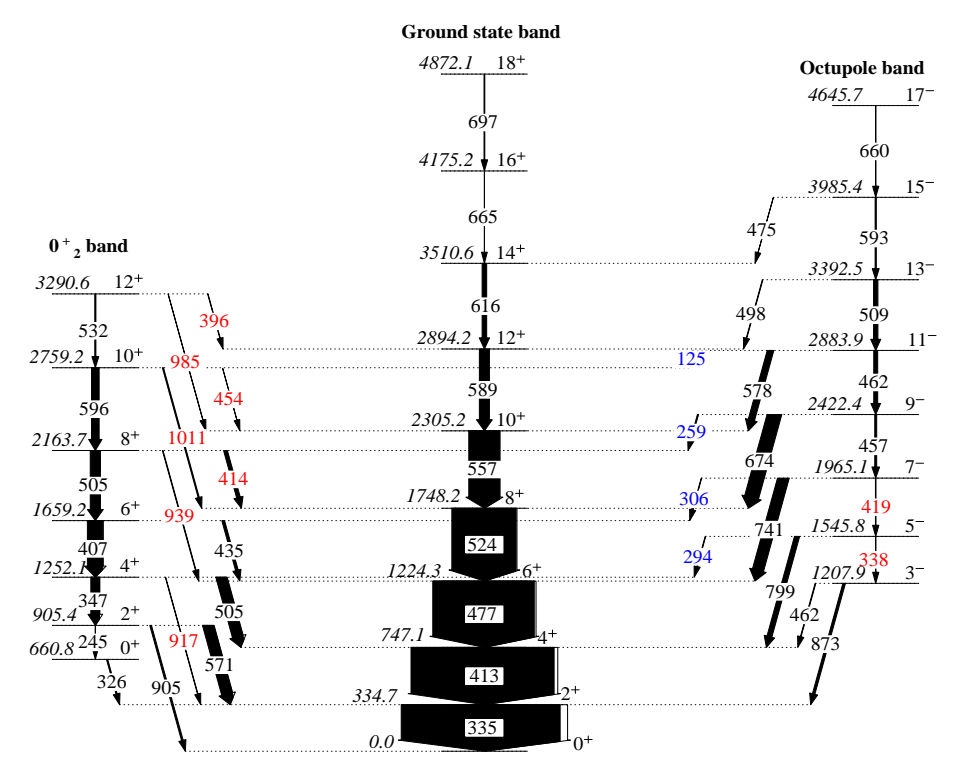

Figure 2: Partial level scheme of ${ }^{154} \mathrm{Dy}$ obtained from ${ }^{155} \mathrm{Gd}\left({ }^{3} \mathrm{He}, 4 \mathrm{n}\right)$ reaction at $37.5 \mathrm{MeV}$ showing the ground state, band built on $0_{2}^{+}, S$ band and the octupole band. New transitions are shown in red and E1 transitions from the octupole band to the band built on $0_{2}^{+}$are shown in blue.

Fig. 2 shows the partial level scheme of ${ }^{154}$ Dy obtained from our experiment. The band built on $\mathrm{O}_{2}^{+}$state which was observed earlier [10] and additional intra-band transitions between this band and the ground state band have been observed. Two in-band E2 transitions have been observed to form the lowest lying negative parity band which is now built on $3^{-}$state. The new two in-band $\gamma$ 
rays connect level $7^{-} \longrightarrow 5^{-}$and $5^{-} \longrightarrow 3^{-}$respectively. These levels were reported in National Nuclear Data Center [11] database but the two $\gamma$ transitions them were not observed. We observe four new E1 transitions from the lowest lying negative parity band to the first excited $0^{+}$band. The $\gamma$ rays observed are $125 \mathrm{keV} 11^{-} \longrightarrow 10^{+}, 259 \mathrm{keV}$ from $9^{-} \longrightarrow 8^{+}, 306 \mathrm{keV}$ from $7^{-} \longrightarrow 6^{+}$, and $294 \mathrm{keV}$ from $5^{-} \longrightarrow 4^{+}$respectively.
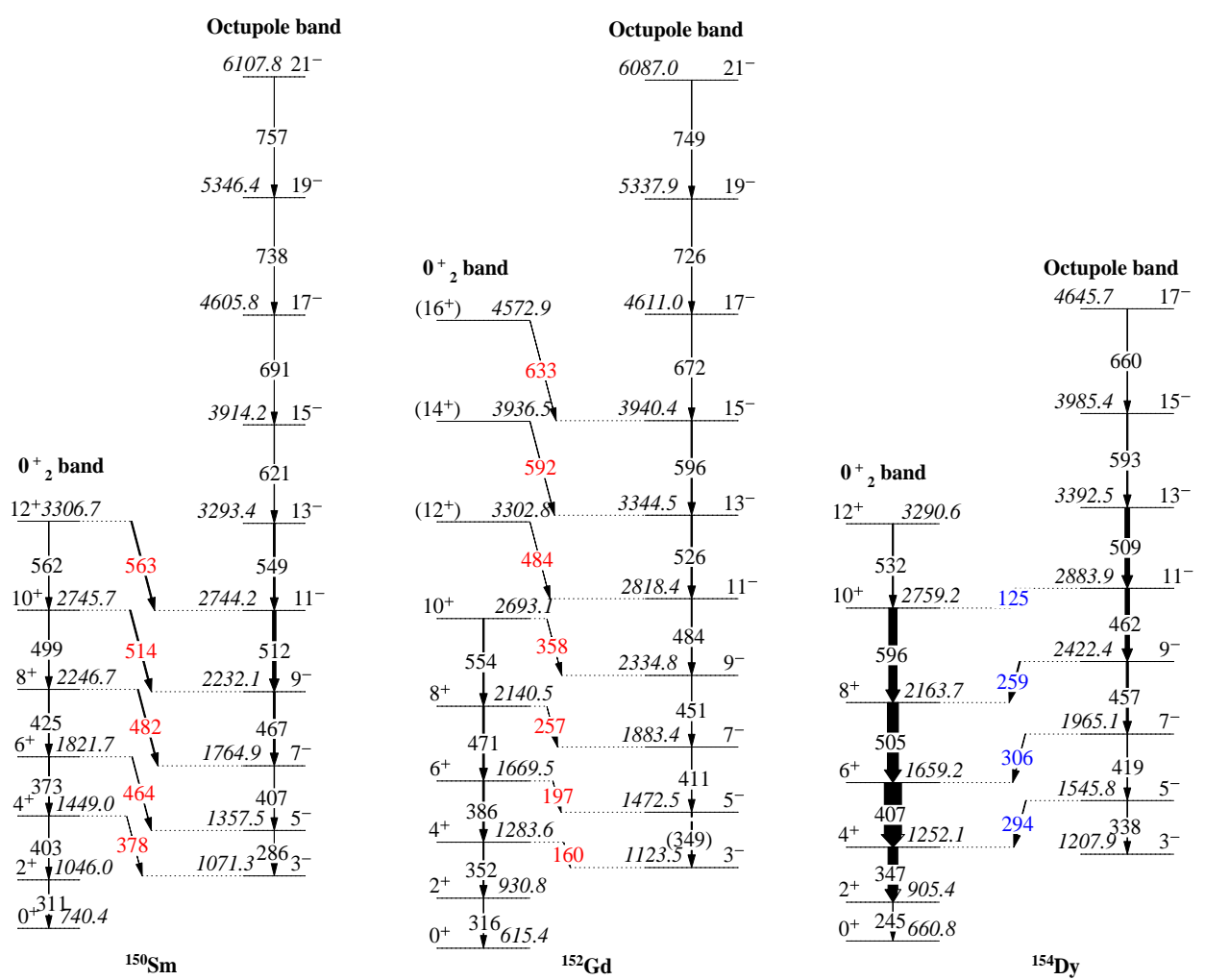

Figure 3: (Red) E1 transitions from $0_{2}^{+} \longrightarrow 33_{1}^{-}$in ${ }^{152} \mathrm{Gd}$ and ${ }^{150} \mathrm{Sm}$ [2] and (Blue) the first observation of E1 transitions from $3_{1}^{-} \longrightarrow 0_{2}^{+}$observed in ${ }^{154}$ Dy.

E1 transitions were observed in ${ }^{150} \mathrm{Sm}$ and ${ }^{152} \mathrm{Gd}$ by Bvumbi et al [2] studying the octupole correlations in $\mathrm{N}=88$ isotones. In $\mathrm{N}=88{ }^{150} \mathrm{Sm}$ and ${ }^{152} \mathrm{Gd}$ these $\mathrm{E} 1$ transitions were observed from the first excited $0_{2}^{+}$band to the lowest lying negative parity [2]. In ${ }^{154} \mathrm{Dy}$, we observed for the first time E1 transitions from the lowest lying negative parity band to the excited $0_{2}^{+}$pairing isomer band. These transitions between the two band suggest that the bands are structurally related to each other. Fig. 3 shows the E1 transitions in ${ }^{154} \mathrm{Dy},{ }^{150} \mathrm{Sm}$ and ${ }^{152} \mathrm{Gd}$. In even-even nuclei 
the negative-parity sequence is shifted up with respect to the positive-parity levels as a function of proton number, this is also illustrated in Fig. 3.

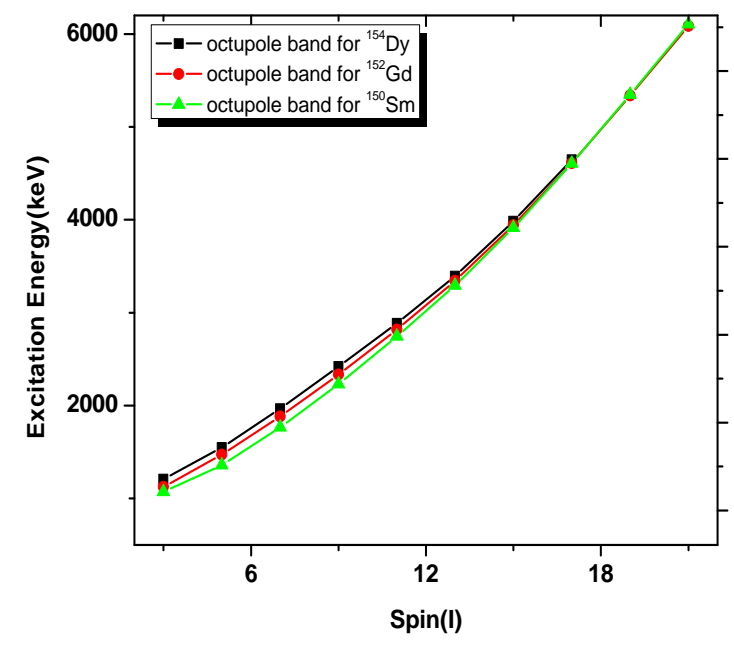

Figure 4: Plot of the level excitation energies against spin for the octupole bands in ${ }^{150} \mathrm{Sm},{ }^{152} \mathrm{Gd}$ and ${ }^{154} \mathrm{Dy}$.

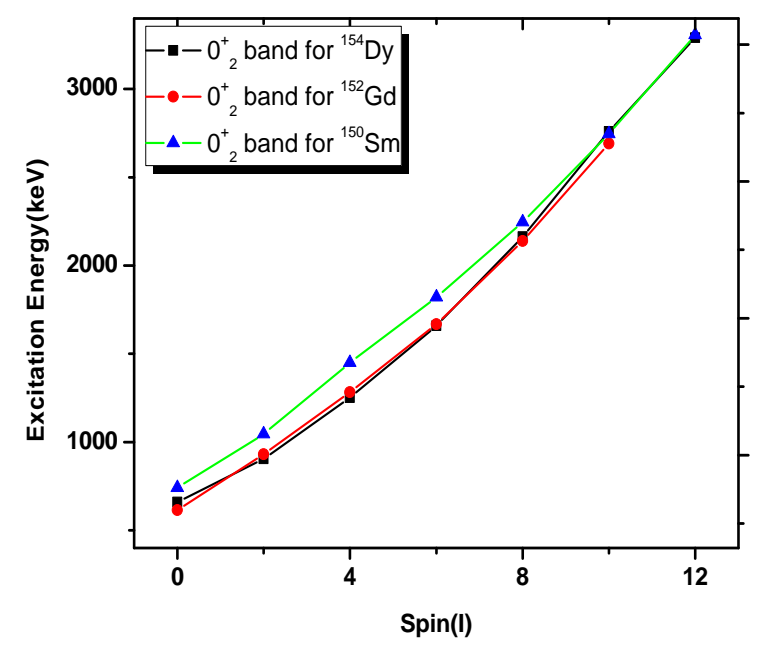

Figure 5: Plot of the level excitation energies against spin for the first excited $0^{+}$band in ${ }^{150} \mathrm{Sm},{ }^{152} \mathrm{Gd}$ and ${ }^{154}$ Dy.

Fig. 4 shows the plots of excitation energy against spin for octupole bands in ${ }^{154} \mathrm{Dy},{ }^{150} \mathrm{Sm}$ and ${ }^{152} \mathrm{Gd}$. This shows that the octupole band in ${ }^{154} \mathrm{Dy}$ is at higher excitation energy than ${ }^{150} \mathrm{Sm}$ and ${ }^{152} \mathrm{Gd}$ up to around spin $17 \hbar$ when all the bands have almost similar excitation energy. Fig. 5 shows that the excitation energy of the first excited $0^{+}$band in ${ }^{154}$ Dy lies between the first excited $0^{+}$band in ${ }^{150} \mathrm{Sm}$ and ${ }^{152} \mathrm{Gd}$. The band built on $0_{2}^{+}$in ${ }^{154} \mathrm{Dy}$ has a unexpected characteristic when compared to first excited $0^{+}$bands in ${ }^{154} \mathrm{Dy},{ }^{150} \mathrm{Sm}$ as seen from Fig. 5 . 


\section{Conclusion}

The presence of these E1 transitions from the lowest lying negative parity band to the first excited $0^{+}$and the discoveries in light actinide nuclei [12] of level patterns similar to rotational bands in reflection asymmetric molecules suggest that some nuclei such as ${ }^{154} \mathrm{Dy}$ at low spin have stable shapes which may incorporate octupole deformation.

We observed in-band E2 transitions connecting negative parity levels therefore in the process establishing the lowest lying negative party band hence the question of ${ }^{154}$ Dy being associated with tetrahedral symmetries [4] is not founded. We have shown the presence of E1 transitions from the lowest lying negative parity band and to the excited $0_{2}^{+}$pairing isomer band. We suggest that our observation of these E1 transitions in ${ }^{154}$ Dy are due to the increase in excitation energy of the lowest lying negative band parity and the characteristic of the first excited $0^{+}$band as seen in Fig. 5 .

\section{Acknowledgments}

We would like to thank the AFRODITE group for their participation in the experiment and for their help during the analysis of the data.

\section{References}

[1] J. F. Sharpey-Schafer, Nucl. Phys. News. Int. 14, 5 (2004).

[2] S. P. Bvumbi et al., Phys. Rev. C87, 044333, (2013).

[3] R. A. Casten and N. V. Zamfir, Phys. Rev. Lett. 471433 (1981).

[4] J. Dudek et al., Phys. Rev. Lett. 88, 252502, (2002).

[5] R. H. Spear and W. N. Catford, Phys. Rev. C41, R1351, (1990).

[6] W. Urban, W. Gast, G. Hebbinghaus, A. Krämer-Flecken, K. P.a Blume, and H. Hübel, Phys. Lett. B185, 331 (1987).

[7] J. Cresswell and J. Sampson, MTsort manual, University of Liverpool, (2012).

[8] D. C. Radford, Nucl. Meth. A361, 297, (1995).

[9] P. J. Twin, Nucl. Instr. Methods 106, 481, (1973).

[10] W. C. Ma et al., Phys. Rev. C65, 034312, (2002).

[11] http: //www. nndc.bnl. gov/chart/getdataset. jsp?nucleus=154DY\&unc=nds.

[12] J. Fernandez-Niello et al., Nucl. Phys. A391, 221 (1982).

[13] R. R. Chasman, Phys. Rev. Lett. 42630 (1979). 